\title{
On Bers generating functions for first order systems of mathematical physics
}

\author{
Vladislav V. Kravchenko ${ }^{1}$, Marco P. Ramirez T. ${ }^{2}$ \\ ${ }^{1}$ Departamento de Matemáticas, CINVESTAV del IPN, Unidad Querétaro, \\ Libramiento Norponiente No. 2000, Fracc. Real de Juriquilla, \\ Querétaro, Qro. C.P. 76230 MEXICO e-mail: vkravchenko@qro.cinvestav.mx \\ ${ }^{2}$ Escuela de Ingeniería de la Universidad La Salle, \\ Benjamín Franklin No. 47, Col. Condesa, C.P. 06140, México D.F. \\ e-mail: mramirez@lci.ulsa.mx
}

December 28, 2018

\begin{abstract}
Considering one of the fundamental notions of Bers' theory of pseudoanalytic functions the generating pair via an intertwining relation we introduce its generalization for biquaternionic equations corresponding to different first-order systems of mathematical physics with variable coefficients. We show that the knowledge of a generating set of solutions of a system allows one to obtain its different form analogous to the complex equation describing pseudoanalytic functions of the second kind and opens the way for new results and applications of pseudoanalytic function theory. As one of the examples the Maxwell system for an inhomogeneous medium is considered, and as one of the consequences of the introduced approach we find a relation between the time-dependent one-dimensional Maxwell system and hyperbolic pseudoanalytic functions and obtain an infinite system of solutions of the Maxwell system. Other considered examples are the system describing force-free magnetic fields and the Dirac system from relativistic quantum mechanics.
\end{abstract}

\section{Introduction}

Bers' theory of pseudoanalytic functions mainly created in fifties of the last century [2] offers interesting and still not fully explored tools for studying and solving linear elliptic equations in the plane. Recent advances in the theory and its applications [1] show that some abstract constructions proposed by Bers can be made completely explicit and applicable to important equations of mathematical physics. For example, pseudoanalytic formal powers introduced and studied by Bers and later on by many other mathematicians had been obtained 
only in some very special situations which represented a considerable obstacle for a further development of pseudoanalytic function theory. This obstacle has been substantially diminished in a recent work reported in [11 due to the fact that there has been found a method for constructing formal powers explicitly in a much more general situation. Moreover, it was shown that this construction offers a tool for calculating explicitly complete systems of solutions of linear elliptic second-order equations in the plane. This progress together with some other recent developments posed more open questions concerning pseudoanalytic function theory, its generalizations and applications. For example, a possibility to develop a hyperbolic pseudoanalytic function theory with applications to hyperbolic equations of mathematical physics was explored in [12] (see also [11] and $[5]$ ). In the present work we use some of the results of $[12$ for obtaining an infinite system of solutions of the one-dimensional time-dependent Maxwell system. Another related important open question is the development of pseudoanalytic function theory in higher dimensions. This was analized in a number of papers (see [14, 10, 1] and [11), and it is clear that in this direction the development is barely starting.

The aim of the present paper is to show that some fundamental ideas of pseudoanalytic function theory are valid in a general situation and applicable to linear systems of mathematical physics both elliptic and hyperbolic. The starting point of Bers' theory is the concept of a generating pair which in a sense means a substitution of a pair of "rectilinear elements" of the plane 1 and $i$ by a pair of quite arbitrary "curvilinear elements" - a pair of complex functions $F$ and $G$ which only should enjoy the property of independence in the sense that any complex function $w$ can be represented in the form $w=\varphi F+\psi G$ where $\varphi$ and $\psi$ are real-valued functions. Beginning with generalizations of the first definitions from analytic function theory like the derivative, Bers shows that behind a generating pair there is always a corresponding generalized CauchyRiemann system which is usually called the Vekua or Carleman-Vekua equation. The knowledge of a generating pair for a Vekua equation allows one to represent it in another form which is the equation for pseudoanalytic functions of the second kind. This form is very convenient for introducing a simple formula for calculation of the $(F, G)$-derivative in the sense of Bers and of the corresponding antiderivative and in fact represents a cornerstone of all further constructions of pseudoanalytic function theory including formal powers.

In this work we show that the concept of a generating set of functions in the sense of Bers is much more universal and can be introduced in relation with first-order systems of mathematical physics with the aid of hypercomplex algebraic tools. This allows one to obtain another form of a corresponding system analogous to that describing pseudoanalytic functions of the second kind. In order to generalize the concept of a generating pair it resulted to be fruitful to develop a slightly different approach to this concept via a certain intertwining relation. We consider it in the next section. Another tool implemented in this paper is the algebra of biquaternions. The related notations are introduced also in section 2

We consider only few examples in this paper chosen in such a way that from 
one side it becomes clear that our approach is general and is applicable to a wide variety of systems and from the other it can be seen that for each particular physically meaningful system some special interesting phenomena may occur. Thus, we consider the time-dependent Maxwell system for inhomogeneous media (section 3), the system describing force-free magnetic fields (section 4) and the Dirac equation (section 5). In the case of the Maxwell system we introduce a generating set of solutions, with its aid we obtain the Maxwell system in the form of an equation for pseudoanalytic functions of the second kind. This allows us to find a relation of the Maxwell system in the one-dimensional case with the hyperbolic pseudoanalytic function theory developed in [12. As a direct consequence of this relation we obtain an infinite system of solutions of the Maxwell equations.

In the case of force-free magnetic fields we show that in fact one exact solution is sufficient to obtain a corresponding generating quartet and to write down the second-kind equation. This interesting phenomenon leads to an observation regarding the quotients of solutions. Namely, we obtain a differential equation satisfied by the quotients of solutions. This result seems to be new even in the case of monogenic functions (a special case when the proportionality factor in the considered system vanishes identically). Finally, in section 5 we show that the concept of a generating set is applicable to the Dirac system with electromagnetic and scalar potentials, and in this case as well the system can be written in another form corresponding to pseudoanalytic functions of the second kind.

\section{Preliminaries}

\subsection{Biquaternions}

We will denote the algebra of biquaternions or complex quaternions by $\mathbb{H}(\mathbb{C})$ with the standard basic quaternionic units denoted by $e_{0}=1, e_{1}, e_{2}$ and $e_{3}$. The complex imaginary unit is denoted by $i$ as usual. The set of purely vectorial quaternions $q=\mathbf{q}$ is identified with the set of three-dimensional vectors.

The quaternionic conjugation of a biquaternion $q=q_{0}+\mathbf{q}$ will be denoted as

$$
\bar{q}=q_{0}-\mathbf{q},
$$

and by $q^{*}$ we denote the complex conjugation of $q$,

$$
q^{*}=\operatorname{Re} q-i \operatorname{Im} q .
$$

Sometimes the following notation for the operator of multiplication from the right-hand side will be used

$$
M^{p} q=q \cdot p .
$$

The main quaternionic differential operator introduced by Hamilton himself and sometimes called the Moisil-Theodoresco operator is defined on continuously 
differentiable biquaternion-valued functions of the real variables $x_{1}, x_{2}$ and $x_{3}$ according to the rule

$$
D q=\sum_{k=1}^{3} e_{k} \partial_{k} q,
$$

where $\partial_{k}=\frac{\partial}{\partial x_{k}}$.

\subsection{Pseudoanalytic functions}

In this subsection we introduce some basic concepts from Bers' theory of pseudoanalytic functions 2 2 and give a slightly varied interpretation of the notion of a generating pair. Precisely this different interpretation allows us to introduce the generating sets for the considered in the subsequent sections systems of mathematical physics.

According to [2] a pair of arbitrary continuously differentiable with respect to the real variables $x$ and $y$ complex-valued functions $F$ and $G$ satisfying the inequality

$$
\operatorname{Im}(\bar{F} G)>0
$$

in a domain $\Omega \subset \mathbb{C}$ is called a generating pair. This inequality means that $F$ and $G$ are independent in the sense that any complex function $W$ defined in $\Omega$ can be expressed in the form

$$
W=\varphi F+\psi G
$$

where $\varphi$ and $\psi$ are real-valued functions.

For a fixed generating pair and in the case when $\varphi$ and $\psi$ are continuously differentiable one can define the $(F, G)$-derivative of the function $W$ in the following way

$$
\stackrel{\circ}{W}=F \partial_{z} \varphi+G \partial_{z} \psi,
$$

where $\partial_{z}=\frac{1}{2}\left(\partial_{x}-i \partial_{y}\right)$. The derivative $\stackrel{\circ}{W}$ exists iff the equality

$$
F \partial_{\bar{z}} \varphi+G \partial_{\bar{z}} \psi=0
$$

holds. Here $\partial_{\bar{z}}=\frac{1}{2}\left(\partial_{x}+i \partial_{y}\right)$.

Introducing the notation

$$
a=-\frac{\bar{F} \partial_{\bar{z}} G-\bar{G} \partial_{\bar{z}} F}{F \bar{G}-\bar{F} G}, \quad b=\frac{F \partial_{\bar{z}} G-G \partial_{\bar{z}} F}{F \bar{G}-\bar{F} G},
$$

equation (2) can be written in the form of a Vekua equation

$$
\partial_{\bar{z}} W-a W-b \bar{W}=0 .
$$

Functions $a$ and $b$ are known as characteristic coefficients of the generating pair $(F, G)$ and solutions of (3) are known as pseudoanalytic functions, or more exactly $(F, G)$-pseudoanalytic functions of the first kind. Solutions of the corresponding equation (2) regarded as complex-valued functions $w=\varphi+i \psi$ are 
called $(F, G)$-pseudoanalytic functions of the second kind. Note that by construction both generating functions $F$ and $G$ are $(F, G)$-pseudoanalytic of the first kind.

Now let us consider the operator from Vekua equation (3), $\partial_{\bar{z}}-a-b C$ where by $C$ we denote the operator of complex conjugation. Take an arbitrary real-valued function $\varphi$ and consider the equality

$$
\left(\partial_{\bar{z}}-a-b C\right)(\varphi f)=f \partial_{\bar{z}} \varphi
$$

where $f$ is some complex function. It is easy to see that this equality holds for any real-valued $\varphi$ iff $f$ is a particular solution of (3). In order to be able to consider a general solution of (3) another particular solution, say, $g$ is needed. In this case we can look for solutions of (3) in the form $W=\varphi f+\psi g$ where $\varphi$ and $\psi$ are real-valued functions and $f$ and $g$ are particular solutions of (3) if only $f$ and $g$ are independent in the sense explained above. Thus we arrive at the concept of a generating pair for a Vekua equation via the intertwining relation (4). We have then

$$
\left(\partial_{\bar{z}}-a-b C\right)(\varphi f+\psi g)=f \partial_{\bar{z}} \varphi+g \partial_{\bar{z}} \psi
$$

which in particular gives us a relation between equations (3) and (2).

\section{Generating sets of solutions for the Maxwell system in inhomogeneous media}

Let us consider the Maxwell equations for inhomogeneous media

$$
\begin{gathered}
\operatorname{rot} \mathbf{H}=\varepsilon \partial_{t} \mathbf{E}+\mathbf{j}, \\
\operatorname{rot} \mathbf{E}=-\mu \partial_{t} \mathbf{H}, \\
\operatorname{div}(\varepsilon \mathbf{E})=\rho, \\
\operatorname{div}(\mu \mathbf{H})=0 .
\end{gathered}
$$

Here $\varepsilon$ and $\mu$ are real-valued functions of coordinates, $\mathbf{E}$ and $\mathbf{H}$ are real-valued vector fields depending on $t$ and spatial variables, the real-valued scalar function $\rho$ and the real vector function $\mathbf{j}$ characterize the distribution of sources of the electromagnetic field.

The wave propagation velocity will be denoted by $c=\frac{1}{\sqrt{\varepsilon \mu}}$, the refraction index by $n=\sqrt{\varepsilon \mu}$ and the intrinsic impedance of the medium by $Z=\sqrt{\frac{\mu}{\varepsilon}}$. As was shown in [7, [8], introducing the notations

$$
\mathbf{c}=\frac{\operatorname{grad} \sqrt{c}}{\sqrt{c}}, \quad \mathbf{Z}=\frac{\operatorname{grad} \sqrt{Z}}{\sqrt{Z}} \text { and } \mathbf{V}=\sqrt{\varepsilon} \mathbf{E}+i \sqrt{\mu} \mathbf{H}
$$

one can rewrite system (5)-(8) in the form of a single biquaternionic equation

$$
\left(\frac{1}{c} \partial_{t}+i D\right) \mathbf{V}-M^{i \mathbf{c}} \mathbf{V}-M^{i \mathbf{Z}} \mathbf{V}^{*}=-\left(\sqrt{\mu} \mathbf{j}+\frac{i \rho}{\sqrt{\varepsilon}}\right)
$$


which in a sourceless situation becomes

$$
\left(\frac{1}{c} \partial_{t}+i D\right) \mathbf{V}-M^{i \mathbf{c}} \mathbf{V}-M^{i \mathbf{Z}} \mathbf{V}^{*}=0 .
$$

Let $\varphi$ be a real-valued function. Then it is easy to see that the equality

$$
\left(\frac{1}{c} \partial_{t}+i D-M^{i \mathbf{c}}-M^{i \mathbf{Z}} C\right)[\varphi \mathbf{V}]=\left(\frac{1}{c} \partial_{t}+i D\right)[\varphi] \cdot \mathbf{V}
$$

holds iff $\mathbf{V}$ is a solution of (9).

Assume that $\left\{\mathbf{V}_{1}, \ldots, \mathbf{V}_{6}\right\}$ are solutions of (9) independent in the sense that for any complex vector function $\mathbf{V}$ there exist real valued functions $\varphi_{k}$, $k=1,2, \ldots, 6$ such that $\mathbf{V}=\sum_{k=1}^{6} \varphi_{k} \mathbf{V}_{k}$. This can be easily written as a condition on a corresponding determinant of a matrix formed by components of $\mathbf{V}_{k}$. Then due to (10) we have that $\mathbf{V}=\sum_{k=1}^{6} \varphi_{k} \mathbf{V}_{k}$ is a solution of (9) if and only if

$$
\sum_{k=1}^{6}\left(\frac{1}{c} \partial_{t}+i D\right)\left[\varphi_{k}\right] \cdot \mathbf{V}_{k}=0
$$

This equation for real-valued functions $\varphi_{k}, k=1,2, \ldots, 6$ is a Bers' equation for Maxwell pseudoanalytic functions of the second kind.

Notice that in a frequently encountered in practice case $\mu=$ Const we have that $\mathbf{c}=\mathbf{Z}$ and (9) turns into the equation

$$
\left(\frac{1}{c} \partial_{t}+i D\right) \mathbf{V}-i\left(\mathbf{V}+\mathbf{V}^{*}\right) \mathbf{c}=0
$$

for which it is easy to propose a triplet of independent solutions in the form

$$
\mathbf{V}_{4}=i e_{1}, \quad \mathbf{V}_{5}=i e_{2}, \quad \mathbf{V}_{6}=i e_{3}
$$

corresponding to a constant magnetic field. Thus, in order to rewrite Maxwell's system in the form (11) it is sufficient to find another triplet of solutions. In some cases this can be done relatively easy. Let us consider a stratified medium, that is $\varepsilon=\varepsilon(x)$ and hence $\mathbf{c}=c_{1}(x) e_{1}=\frac{c \prime(x)}{2 c(x)} e_{1}$. Then the vectors

$$
\mathbf{V}_{1}=c e_{1}, \quad \mathbf{V}_{2}=\frac{e_{2}}{c}, \quad \text { and } \mathbf{V}_{3}=\frac{e_{3}}{c}
$$

are solutions of (12). Consequently, the Maxwell system in this case is equivalent to equation (11) (for Maxwell pseudoanalytic functions of the second kind):

$$
\left(\frac{1}{c} \partial_{t}+i D\right) \varphi_{1} \cdot c e_{1}+\sum_{k=2}^{3}\left(\frac{1}{c} \partial_{t}+i D\right) \varphi_{k} \cdot \frac{e_{k}}{c}+\sum_{k=4}^{6}\left(\frac{1}{c} \partial_{t}+i D\right) \varphi_{k} \cdot i e_{k-3}=0 .
$$

For a better understanding of this equation as well as of equation (12) let us consider solutions depending on $t$ and $x$ only:

$$
\left(\frac{1}{c(x)} \partial_{t}+i e_{1} \partial_{x}\right) \mathbf{V}(t, x)-i\left(\mathbf{V}(t, x)+\mathbf{V}^{*}(t, x)\right) c_{1}(x) e_{1}=0
$$


One can observe that equations for $V_{1}$ and those for $V_{2}, V_{3}$ are not coupled. We have

$$
\left(\frac{1}{c} \partial_{t}+i e_{1} \partial_{x}\right) V_{1}-i\left(V_{1}+V_{1}^{*}\right) c_{1} e_{1}=0
$$

and

$$
\left(\frac{1}{c} \partial_{t}+i e_{1} \partial_{x}\right)\left(V_{2} e_{2}+V_{3} e_{3}\right)-i\left(\left(V_{2}+V_{2}^{*}\right) e_{2}+\left(V_{3}+V_{3}^{*}\right) e_{3}\right) c_{1} e_{1}=0 .
$$

The first of these equations can be easily solved. Note that $\partial_{t} V_{1} \equiv 0$ and $\partial_{x} V_{1}-2 c_{1} \operatorname{Re} V_{1}=0$. That is $\operatorname{Im} V_{1} \equiv$ Const and $\partial_{x} \operatorname{Re} V_{1}-\frac{c \prime(x)}{c(x)} \operatorname{Re} V_{1}=0$ which gives us a general form of the component $V_{1}$ in the case under consideration, $V_{1}=a_{1} c(x)+i a_{2}$ where $a_{1}$ and $a_{2}$ are arbitrary real constants.

Now let us consider equation (14). It can be written as the following bicomplex equation

$$
\left(\frac{1}{c} \partial_{t}+i e_{1} \partial_{x}\right) \Phi+i c_{1} e_{1}\left(\Phi+\Phi^{*}\right)=0
$$

for the bicomplex function $\Phi=V_{2}+V_{3} e_{1}$.

Denote by $N$ an antiderivative of the refraction index $n$ and consider the following change of the variable $x \mapsto \xi=N(x)$. Then the function $\Psi(t, \xi(x))=$ $\Phi(t, x)$ as a function of the variables $t$ and $\xi$ satisfies the following equation

$$
\left(\partial_{t}+i e_{1} \partial_{\xi}\right) \Psi(t, \xi)+i e_{1} \frac{C^{\prime}(\xi)}{2 C(\xi)}\left(\Psi(t, \xi)+\Psi^{*}(t, \xi)\right)=0
$$

where $C(\xi(x))=c(x)$. Note that introducing a new unity $j=i e_{1}$ which obviously satisfies the equality $j^{2}=1$ and considering the bicomplex function in the form $\Psi=\Psi_{1}+\Psi_{2} j$ where $\Psi_{1,2}=u_{1,2}+v_{1,2} e_{1}$ with $u_{1,2}$ and $v_{1,2}$ being real valued functions we can rewrite the last equation as follows

$$
\left(\partial_{t}+j \partial_{\xi}\right) \Psi(t, \xi)+j \frac{C^{\prime}(\xi)}{2 C(\xi)}\left(\Psi(t, \xi)+\Psi^{*}(t, \xi)\right)=0
$$

where $\Psi^{*}=\Psi_{1}-\Psi_{2} j$. Finally, applying the conjugation operator and introducing the new bicomplex function $W=\sqrt{C} \Psi^{*}$ we arrive at the equation

$$
\frac{1}{2}\left(\partial_{\xi}-j \partial_{t}\right) W-\frac{f^{\prime}(\xi)}{2 f(\xi)} W^{*}=0
$$

where $f=\sqrt{C}$, which can be written in the form of a hypebolic Vekua equation

$$
\partial_{\bar{z}} W-\frac{f_{\bar{z}}}{f} \bar{W}=0
$$

where $\partial_{\bar{z}}=\frac{1}{2}\left(\partial_{\xi}-j \partial_{t}\right)$ and instead of an asterisk we used bar for denoting the same conjugation with respect to $j$. This equation in the case when $W$ has values in the algebra of hyperbolic numbers which we denote as $\mathcal{H}$, that is when $W=W_{1}+W_{2} j$ with $W_{1}$ and $W_{2}$ being real valued, was introduced and studied in [12] and [1] in relation to the Klein-Gordon equation and in [5] in relation to 
the Zakharov-Shabat system. Notice that due to the fact that $f$ is real valued, equation (15) in fact consists of two separate equations for $\mathcal{H}$-valued functions, that is it reduces to a pair of equations which were considered in the previous publications [5, 11] and [12. In order to observe this one needs to write the function $W$ in the form $W=w_{1}+w_{2} e_{1}$ where $w_{1}$ and $w_{2}$ are $\mathcal{H}$-valued. Then equation (15) is equivalent to the following pair of separate equations

$$
\partial_{\bar{z}} w_{1}-\frac{f_{\bar{z}}}{f} \overline{w_{1}}=0 \quad \text { and } \quad \partial_{\bar{z}} w_{2}-\frac{f_{\bar{z}}}{f} \overline{w_{2}}=0 .
$$

Now gathering all the introduced transformations we have that

$$
w_{1}(t, \xi)=\sqrt{C(\xi)}\left(\sqrt{\widetilde{\varepsilon}(\xi)} \widetilde{E}_{2}(t, \xi)-\sqrt{\mu} \widetilde{H}_{3}(t, \xi) j\right)
$$

and

$$
w_{2}(t, \xi)=\sqrt{C(\xi)}\left(\sqrt{\widetilde{\varepsilon}(\xi)} \widetilde{E}_{3}(t, \xi)+\sqrt{\mu} \widetilde{H}_{2}(t, \xi) j\right)
$$

where tilde means that the corresponding original function was written as a function of $\xi$, e.g., $\widetilde{\varepsilon}(\xi(x))=\varepsilon(x)$.

Thus, all theory developed in [12] and [11 is applicable in this case to the hyperbolic pseudoanalytic functions $w_{1}$ and $w_{2}$. As an interesting application let us mention a possibility to construct an infinite system of exact solutions of (15) using the results from [12] and [11] combined with the elegant formulas obtained by Bers and Gelbart in the elliptic case [2] Namely, we notice that the pair of functions

$$
(F, G)=(f, j / f)
$$

is a generating pair for both equations (16) and moreover it has a form convenient for applying the results of Bers and Gelbart. Following [2] (see [11, Sect. 4.2] for the slightly corrected formulas), we give explicit formulas for the formal powers corresponding to the generating pair (17) with $f$ depending on one Cartesian variable $\xi$. For simplicity we assume that $z_{0}=0$ and $F(0)=1$. In this case the formal powers are constructed in an elegant manner as follows. First, denote

$$
X^{(0)}(\xi)=\tilde{X}^{(0)}(\xi)=1
$$

and for $n=1,2, \ldots$ denote

$$
X^{(n)}(\xi)= \begin{cases}n \int_{0}^{\xi} X^{(n-1)}(x) \frac{d x}{f^{2}(x)} & \text { for an odd } n \\ n \int_{0}^{\xi} X^{(n-1)}(x) f^{2}(x) d x & \text { for an even } n\end{cases}
$$




$$
\widetilde{X}^{(n)}(\xi)= \begin{cases}n \int_{0}^{\xi} \widetilde{X}^{(n-1)}(x) f^{2}(x) d x & \text { for an odd } n \\ n \int_{0}^{\xi} \widetilde{X}^{(n-1)}(x) \frac{d x}{f^{2}(x)} & \text { for an even } n\end{cases}
$$

Then for $a=a^{\prime}+j a^{\prime \prime}, a^{\prime}, a^{\prime \prime} \in \mathbb{R}$ and $z=\xi+t j$ we have

$$
Z^{(n)}(a, 0, z)=f(\xi) \operatorname{Re}_{*} Z^{(n)}(a, 0, z)+\frac{j}{f(\xi)} \operatorname{Im}_{*} Z^{(n)}(a, 0, z)
$$

where

$$
\begin{aligned}
* Z^{(n)}(a, 0, z) & =a^{\prime} \sum_{m=0}^{n}\left(\begin{array}{c}
n \\
m
\end{array}\right) X^{(n-m)} j^{m} t^{m} \\
& +j a^{\prime \prime} \sum_{m=0}^{n}\left(\begin{array}{c}
n \\
m
\end{array}\right) \widetilde{X}^{(n-m)} j^{m} t^{m} \quad \text { for an odd } n
\end{aligned}
$$

and

$$
\begin{aligned}
* Z^{(n)}(a, 0, z) & =a^{\prime} \sum_{m=0}^{n}\left(\begin{array}{c}
n \\
m
\end{array}\right) \widetilde{X}^{(n-m)} j^{m} t^{m} \\
& +j a^{\prime \prime} \sum_{j=0}^{n}\left(\begin{array}{c}
n \\
m
\end{array}\right) X^{(n-m)} j^{m} t^{m} \quad \text { for an even } n .
\end{aligned}
$$

For any $a \in \mathcal{H}$ and $n \in \mathbb{N}$ the formal power $Z^{(n)}(a, 0, z)$ is a solution of (16). Thus, the system of constructed formal powers gives an infinite system of solutions of the Maxwell equations in the case under consideration. Up to now it is an open question what part of the kernel of (16) and consequently of the Maxwell system (14) can be approximated by the obtained exact solutions.

\section{Generating solution for force-free magnetic fields}

The system describing force-free magnetic fields has the form

$$
\begin{gathered}
\operatorname{rot} \mathbf{B}+\alpha \mathbf{B}=0, \\
\operatorname{div} \mathbf{B}=0,
\end{gathered}
$$

where $\mathbf{B}$ is a vector describing the magnetic field and the commonly known as proportionality factor $\alpha$ is a real-valued function of spatial variables. This system is of great importance in such fields as high temperature superconductors 
and dynamics of magnetofluids (see e.g. [4], [15], [16]). It has been analized by different methods including those of quaternionic analysis (see [3], [13] for the theory in the case when $\alpha$ is a constant and [8], [9] and [17] for some developments in a nonconstant case).

Obviously system (20) can be written in the form of a quaternionic equation

$$
(D+\alpha) \mathbf{B}=0
$$

which is completely equivalent to (20). In what follows we do not restrict ourselves by purely vectorial null solutions of the operator $D+\alpha$ and consider the equation

$$
(D+\alpha) B=0
$$

on the class of continuously differentiable $\mathbb{H}(\mathbb{C})$-valued functions $B$ with the proportionality factor $\alpha$ being a complex-valued function. Applying the same idea as before we find out that for any scalar (complex-valued) function $\varphi \in$ $C^{1}(\Omega)$ where $\Omega$ is some domain in $\mathbb{R}^{3}$ the equality

$$
(D+\alpha)(\varphi b)=(D \varphi) b
$$

holds with $b$ being an $\mathbb{H}(\mathbb{C})$-valued function iff $b$ is a solution of (21). Thus, chosing four independent solutions of (21) $b_{k}, k=\overline{0,3}$ we can look for a general solution of (21) in the form $B=\sum_{k=0}^{3} \varphi_{k} b_{k}$ where the scalar functions $\varphi_{k}$ should satisfy the equation

$$
\sum_{k=0}^{3} D \varphi_{k} \cdot b_{k}=0
$$

which is an analogue of the Bers equation for pseudoanalytic functions of the second kind.

Nevertheless one can notice that in the case of equation (21) in fact it is sufficient to have only one particular solution $b$. As $b \lambda$ with $\lambda$ being a constant biquaternion is also a solution of (21) the generating quartet $b_{k}, k=\overline{0,3}$ can be proposed in the form $b_{k}=b e_{k}$ if only $b$ is invertible in any point of the domain of interest $\Omega$.

Thus, we obtain the following statement.

Proposition 1 Let $b$ be a particular solution of (21), invertible in any point of $\Omega \subset \mathbb{R}^{3}$. Then the general solution of (21) can be represented as the product $B=b \Phi$ where the components of the $\mathbb{H}(\mathbb{C})$-valued function $\Phi$ satisfy the equation

$$
\sum_{k=0}^{3} D \Phi_{k} \cdot b e_{k}=0
$$

Proof. Under the conditions of the proposition let us consider the function $B=b \Phi=\sum_{k=0}^{3} b \Phi_{k} e_{k}$. Now application of the operator $D+\alpha$ to $B$ gives $(D+\alpha) B=\sum_{k=0}^{3} D \Phi_{k} \cdot b e_{k}+\sum_{k=0}^{3} \Phi_{k} \cdot((D+\alpha) b) e_{k}$ from where it is seen that $B$ is a solution of (21) iff (23) is valid. 
Remark 2 It should be noticed that the last proposition is also valid when $\alpha$ is a full biquaternionic function.

From the above proposition the following interesting fact about the quotients of solutions of (21) follows.

Proposition 3 Let $f$ and $g$ be solutions of (21) and $f$ be invertible in the domain of interest. Then the function $\Phi=f^{-1} g$ is a solution of the equation

$$
\sum_{k=0}^{3} D \Phi_{k} \cdot f e_{k}=0
$$

and if $g$ is also invertible the inverse $\Psi=\Phi^{-1}=g^{-1} f$ is a solution of the equation

$$
\sum_{k=0}^{3} D \Psi_{k} \cdot g e_{k}=0
$$

Proof. According to the previous proposition if $g$ is a solution of (21) it can be represented in the form $g=f \Phi$ where the components of the biquaternionic function $\Phi=f^{-1} g$ are solutions of (24). Now noticing that if $g$ is invertible as well the function $\Phi$ is also invertible and hence consideration of $f=g \Phi^{-1}$ leads to equation (25).

Remark 4 This proposition is of course also valid in the special case $\alpha \equiv 0$ corresponding to quaternionic monogenic or hyperholomorphic functions. It is well known that in general a quotient of two monogenic functions must not be monogenic. Proposition 3 gives us a precise equation satisfied by the quotient.

\section{The Dirac equation}

We consider here the Dirac equation for one spin 1/2 particle under the influence of an electromagnetic potential, but in fact the procedure is applicable to other kinds of physical potentials (scalar, pseudoscalar, etc.). The Dirac equation has the form

$$
\left(\gamma_{0} \partial_{t}-\sum_{k=1}^{3} \gamma_{k} \partial_{k}+i m+i \phi \gamma_{0}+i \sum_{k=1}^{3} A_{k} \gamma_{k}\right) \Psi=0,
$$

where $\gamma_{0}, \gamma_{1}, \gamma_{2}$ and $\gamma_{3}$ are the Dirac $\gamma$-matrices (see, e.g., [19]), $m$ is the mass of the particle, $\phi$ is the electric potential and $A_{1}, A_{2}$ and $A_{3}$ are components of the magnetic potential $\vec{A}$. The wave function $\Psi$ is a $\mathbb{C}^{4}$-vector function $\Psi=\left(\Psi_{0}, \Psi_{1}, \Psi_{2}, \Psi_{3}\right)$. Equation (26) is considered in some domain $\mathcal{G} \subset \mathbb{R}^{4}$.

As was shown in [6] (see also [13] and [8]) the Dirac equation (26) can be written in the following quaternionic form

$$
\left(D-\partial_{t} M^{e_{1}}+\mathbf{a}-M^{i \phi e_{1}+m e_{2}}\right) \Phi=0
$$


where the purely vectorial quaternion $\mathbf{a}$ is obtained from the magnetic potential $\vec{A}$, and the $\mathbb{H}(\mathbb{C})$-valued function $\Phi$ is related to $\Psi$ by an invertible matrix transformation (see [13], 8] and [1]). It is worth mentioning that this form of the Dirac equation was recently rediscovered in 18 .

Consideration of solutions of (26) with fixed energy $\Psi(t, \mathbf{x})=\Psi_{\omega}(\mathbf{x}) e^{i \omega t}$ leads to the biquaternionic equation

$$
\left(D+\mathbf{a}+M^{\mathbf{b}}\right) W=0
$$

where $W$ is an $\mathbb{H}(\mathbb{C})$-valued function of three spatial variables and $\mathbf{b}=-i(\phi+$ $\omega) e_{1}-m e_{2}$.

Proposition 5 Let the biquaternionic functions $F_{0}, F_{1}, F_{2}$ and $F_{3}$ be independen 1 solutions of (27). Then $W=\sum_{k=0}^{3} \varphi_{k} F_{k}$ where $\varphi_{0}, \varphi_{1}, \varphi_{2}$ and $\varphi_{3}$ are scalar functions is a solution of (27) if and only if the following equation is satisfied

$$
\sum_{k=0}^{3}\left(D \varphi_{k}\right) F_{k}=0 .
$$

Obviously, equation (28) is an analogue of equation (2) describing pseudoanalytic functions of the second kind.

\section{Conclusions}

In the present paper it was shown that the concept of a generating pair is not limited to the classical pseudoanalytic function theory and can be introduced in relation with a variety of first-order systems of mathematical physics. Here we considered the Maxwell system for inhomogeneous media, the system describing force-free magnetic fields and the Dirac system from relativistic quantum mechanics. Nevertheless the approach presented in this paper and based on the consideration of a generating solution as an intertwining operator is clearly more general. The knowledge of a generating set of solutions makes it possible to rewrite the original system in a form analogous to the Vekua equation for pseudoanalytic functions of the second kind which opens the way to construction of classes of solutions as was shown in section 3 for the Maxwell system and perhaps more important to the development of Bers' theory for the corresponding system of mathematical physics including such concepts as the derivative, antiderivative, formal powers, Taylor and Laurent series, etc.

\section{References}

[1] P. Berglez, On generalized derivatives and formal powers for pseudoanalytic functions. Matematiche 2007, v. 62, 29-36.

\footnotetext{
${ }^{1}$ In the sense that any $\mathbb{H}(\mathbb{C})$-valued function $W$ can be written as $W=\sum_{k=0}^{3} \varphi_{k} F_{k}$ with $\varphi_{k}$ being scalar functions.
} 
[2] L. Bers, Theory of pseudo-analytic functions. Institute of Mathematics and Mechanics, New York University, New York, 1953.

[3] K. Gürlebeck, W. Sprössig, Quaternionic analysis and elliptic boundary value problems. Berlin: Akademie-Verlag, 1989.

[4] R. D. Kamien, Force free configurations of vortices in high temperature superconductors near the melting transition, Phys. Rev. B, 1998, v. 58, 8218-8221.

[5] V. G. Kravchenko, V. V. Kravchenko and S. Tremblay Zakharov-Shabat system and hyperbolic pseudoanalytic function theory. Mathematical Methods in the Applied Sciences, 2010, published Online First.

[6] V. V. Kravchenko, On a biquaternionic bag model. Zeitschrift für Analysis und ihre Anwendungen, 1995, v. 14, 3-14.

[7] V. V. Kravchenko, Quaternionic equation for electromagnetic fields in inhomogeneous media. In: Progress in Analysis, v. 1, Eds. H. Begehr, R. Gilbert and M. Wah Wong, 361-366, World Scientific, 2003.

[8] V. V. Kravchenko, Applied quaternionic analysis. Research and Exposition in Mathematics Series, Vol. 28, Lemgo: Heldermann Verlag, 2003.

[9] V. V. Kravchenko, On force-free magnetic fields, a quaternionic approach. Mathematical Methods in Applied Sciences, 2005, Vol. 28, 379-386.

[10] V. V. Kravchenko On a factorization of second order elliptic operators and applications. Journal of Physics A: Mathematical and General, 2006, v. 39, 12407-12425.

[11] V. V. Kravchenko Applied pseudoanalytic function theory. Series: Frontiers in Mathematics, Basel: Birkhäuser, 2009.

[12] V. V. Kravchenko, D. Rochon and S. Tremblay On the Klein-Gordon equation and hyperbolic pseudoanalytic function theory. J. Phys. A: Math. Theor. 2008, v. 41, issue 6, 065205.

[13] V. V. Kravchenko and M. V. Shapiro Integral representations for spatial models of mathematical physics. Harlow: Addison Wesley Longman Ltd., Pitman Res. Notes in Math. Series, v. 351, 1996.

[14] H. Malonek, Generalizing the (F,G)-derivative in the sense of Bers. In Clifford Algebras and Their Application in Mathematical Physics, Dordrecht: Kluwer Academic Publishers, 247-257, 1996.

[15] G. E. Marsh, Force free magnetic fields: solutions, topology and applications. World Scientific, 1995. 
[16] M. P. Ramirez T., S. Cruz B., R. Gomez M., On Force-Free Magnetic Fields with non-constant proportionality factor and their applications to High Temperature Superconductors Theory, applying quaternionic analysis, 4th ICEEE 2007, IEEE Catalog Number: 07EX1762C, Library of Congress: 2007923398, p. 393-396, Mexico, 2007.

[17] M. P. Ramirez T., S. Cruz B., R. Gomez M., On Force-Free Magnetic Fields with a non-constant proportionality factor, applying quaternionic analysis. Bulletin de la Societe des Sciences et des Lettres de Lodz, PL ISSN 0459-6854, Poland, Vol. LV, 2009.

[18] Ch. Schwartz Relativistic quaternionic wave equation. Journal of Mathematical Physics, 2006, v. 47, no. 12, 122301.

[19] B. Thaller The Dirac equation. Heidelberg: Springer-Verlag, 1992. 